\title{
TAGUNG
}

\section{Differenzierte Integration im Spannungsfeld von Erwartung und politischer Realität: Eine Bewertung ihrer Auswirkungen auf den europäischen Integrationsprozess}

\author{
Stefanie John*
}

Die Debatte über Gewinne aus und Grenzen einer differenzierten Integration ist aus der europäischen Integrationsgeschichte nicht wegzudenken. Modelle wie Kerneuropa, Avantgarde, Europa à la Carte oder konzentrische Kreise wurden als Zukunftsvisionen, pragmatische Lösungen oder Drohungen in den politischen Diskurs eingeworfen, denen die fundamentale Fragestellung über die Reichweite, den Fortgang oder die praktische Umsetzung der Integration zu Grunde liegen. Vor allem in Situationen, in denen divergierende Vorstellungen, Unterschiede in der politischen Machbarkeit oder im politischen Willen existieren, wird nach alternativen Instrumenten zur Überbrückung dieser Divergenzen gesucht. In der gemeinsamen DreiLänder-Tagung der Schwesterorganisationen der European Community Studies Association (ECSA) aus Österreich, Deutschland und der Schweiz sollte neben einem Resümee über die bisherige Anwendung des Konzepts eine Bewertung der Tragkraft für einzelne Politikbereiche, für die Zusammenarbeit mit Drittstaaten und als Instrument für zukünftige Integrationsschritte vorgenommen werden.

Differenzierte Integration als Instrument für ein Voranschreiten der Integration durch Kooperation, Drohung und Erpressung

Hauptargument der historischen Betrachtung von Wilfried Loth war, dass die Idee eines Kerneuropas keine neue Erfindung, sondern vielmehr ein Merkmal des bisherigen europäischen Integrationsprozesses und der heterogenen Interessenlage der Mitgliedstaaten sei. In

\section{Kerneuropa: Differenzierte Integration als Konzept für die erweiterte EU oder als Grundlage für ihre Spaltung?}

6. Gemeinsame Tagung von ECSA Austria, Arbeitskreis Europäische Integration und ECSA Suisse

Wien, 10. und 11. November 2005

Begrïßung und Einführung

Prof. Dr. Fritz BREUSS, Wirtschaftsuniversität Wien/Österreich, Präsident von ECSA Österreich

Differenzierte Integration in der Geschichte der europäischen Integration

Prof. Dr. Wilfried LOTH, Universität Duisburg-Essen

Kommentar: Prof. Dr. Dieter FREIBURGHAUS, Université de Lausanne/Schweiz

Europäische Politiken I: Der Raum der Freiheit, der Sicherheit und des Rechts

Prof. Dr. Martin BÖSE, Rheinische Friedrich-Wilhelms-Universität Bonn

Europäische Politiken II: Die Wirtschaftsund Währungsunion

Prof. Dr. Fritz BREUSS, Wirtschaftsuniversität Wien/Österreich

Kommentar: Prof. Dr. Richard SENTI, Eidgenössische Technische Hochschule Zürich/Schweiz

* Dipl. Pol. Stefanie John, Lehrstuhl für Internationale Politik, Ruhr-Universität Bochum. 
den 1960er Jahren ging es in erster Linie um die Wahrung der Perspektive einer politischen Union. Hierunter einzuordnen sei De Gaulles Versuch, eine politische Union zu etablieren, die aus dem Kern der sechs Gründerstaaten hervorgehen sollte. In den 1970er Jahren rückten divergierende wirtschaftliche Interessen auf dem Wege zu einer Währungsunion in den Vordergrund. Willy Brandt sah den $\mathrm{Zu}$ sammenhalt der Gemeinschaft aufgrund der Nichtbeteiligung Italiens, Großbritanniens und Irlands an der im März 1973 eingeführten Währungsschlange gefährdet und schlug deshalb vor, dass eine wirtschaftlich starke Pioniergruppe die wirtschaftliche Integration voranbringen solle. In den 1990er Jahren führte die Frustration über die Blockadehaltung von Großbritannien und Dänemark zur Wiederbelebung der Kerneuropa-Diskussion. So sah Loth vor allem in der 1994 vom französischen Premierminister Éduoard Ballardur gestarteten Kerneuropa-Debatte und erweitert um das Schäuble-Lamers-Papier ein strategisches Instrument, die britische Blockade bei der Osterweiterung und weiteren Vertiefungsschritten zu unterlaufen. Dieses Konzept könne nach Loth aber von Mitgliedstaaten, die sich gegen eine weitere Integration stellen, aufgrund seiner inneliegenden Spill-Over-Wirkung nur abgelehnt werden. Neben diesem Drohpotential böte das Kerneuropakonzept aber gleichzeitig Lösungspotentiale für das institutionelle Dilemma, welches mit der Erweiterung der Europäischen Union auf 25 Mitgliedstaaten erwartet wurde. Aufgenommen wurde es von Jacques Delors (1999) mit der Forderung nach einer europäischen Avantgarde und im Plädoyer von Joschka Fischer (Mai 2000) für ein Gravitationszentrum. $\mathrm{Zu}$ Beginn des 21. Jahrhunderts sei vor allem die Angst vor einer Überdehnung der Union die treibende Kraft für das Aufflammen der Debatte. Insgesamt seien die Kerneuropakonzeptionen durchaus als zielführend zu bewerten - sei es für eine partielle Integration oder als glaubhafte Drohung ihrer Verwirklichung.

In seinem Kommentar zweifelte Dieter Freiburghaus an dieser Kraft des Drohpotentials.
Europäische Politiken III: Außenbeziehungen Dr. Mathias JOPP, Institut für Europäische Politik, Berlin; Prof. Dr. Stefan GRILLER, Wirtschaftsuniversität Wien/Österreich Kommentar: Dr. Elisabeth TICHY-FISSLBERGER, Bundesministerium für auswärtige Angelegenheiten, Wien/Österreich

Europäische Politiken IV: Umweltpolitik Prof. Dr. Christian CALLIESS, Georg-August-Universität Göttingen

Kommentar: Dr. Alois LEIDWEIN, Bundesministerium für auswärtige Angelegenheiten, Wien/Österreich, Genf/Schweiz

Übergang oder Dauerzustand? Differenzierte Integration und die Einheitlichkeit der zukünftigen Union

Dr. Franz CROMME, Staatssekretär a.D., Berlin

Kommentar: Stephan KUX, Volkswirtschaftsdirektion des Kantons Zürich/ Schweiz

Diskriminierungsverbot und der Schutz der Wettbewerbsbedingungen: Vertragliche Fesseln der differenzierten Integration Dr. Walter OBWEXER, Leopold-Franzens-Universität Innsbruck/Österreich Kommentar: Dr. Daniel THYM, Humboldt-Universität Berlin

Differenzierte Integration in oder konzentrische Kreise um die EU?

Prof. Dr. Thomas COTTIER, Universität Bern/Schweiz

Kommentar: Dr. Kathrin BLANCK, Bundesministerium für auswärtige Angelegenheiten Wien/Österreich

Differenzierte Integration innerhalb oder außerhalb der EU-Institutionen und die Perspektive einer „Neugründung “ durch eine „Avantgarde“ - Sprengsatz oder unitarische Kraft des Konzepts?

Prof. Dr. Dr. h.c. Peter-Christian MÜLLER-GRAFF, Ruprecht-Karls-Universität Heidelberg

Kommentar: Prof. Dr. Christian JOERGES, Europäisches Hochschulinstitut Florenz/Italien 
Generell kritisierte Freiburghaus den Begriff der differenzierten Integration, da dieser eher als Sammelbegriff funktioniere und deshalb seine analytische Kraft verloren ginge. Einerseits würden sich darunter eine Vielzahl von Konzeptionen (Avantgarde, Europa verschiedener Geschwindigkeiten, harter Kern) verbergen, andererseits sollten damit verschiedene Ziele verfolgt werden (Vertiefung der Integration, aber auch Abgrenzung von bestimmten Staaten oder Zusammenarbeit in Bereichen, in denen die Gemeinschaft keine Kompetenzen besitzt). Allerdings räumte Freiburghaus ein, dass eine reale Differenzierung feststellbar sei, die er anhand dreier Bereiche näher betrachtete: Differenzierung im Verhältnis zu den Nachbarn, bei Beitritten und erpresste Differenzierung. Unter ersterem sei das Verhältnis zu Nachbarn, die nicht oder noch nicht beigetreten sind, zu verstehen. Hier sei eine hohe Flexibilität der Europäischen Union festzustellen, auf die unterschiedlichen Situationen in beitritts(un-) willigen Staaten einzugehen und entsprechende bilaterale Verträge sowie Kooperationen anzubieten. Unter der Differenzierung bei Beitritten erfasste Freiburghaus den unterschiedlichen Integrationswillen der Mitgliedstaaten und dass die Teilnahme aller Staaten nicht erwünscht sei. Als Beispiele dienten das Schengener Abkommen oder die Verteidigungsund Währungspolitik. Sonderstellungen von Schweden, Dänemark und Großbritannien in der Währungspolitik oder Ausnahmen Dänemarks in Politikbereichen der dritten Säule ließen sich jedoch auf Erpressung und nicht auf das Konzept der differenzierten Integration rückführen.

\section{Optimale Bedingungen der Zusammenarbeit im Rahmen des Unionsrechts?}

Aus der juristischen Perspektive von Martin Böse trete differenzierte Integration in zwei verschiedenen Dimensionen auf: einerseits im vertraglichen Rahmen der Europäischen Union und andererseits in Form einer vertieften Zusammenarbeit außerhalb des Unionsrechts. Im ersten Falle sei differenzierte
Integration letztlich eine Abweichung von der allgemeinen Geltung und hauptsächlich in Kernbereichen staatlicher Souveränität vorzufinden. Den Prototyp der differenzierten Integration stelle das Schengener Abkommen dar. Andere Formen böten die Art. 34, 35 und 4045 EUV als verstärkte Zusammenarbeit. Allerdings sei dieses Instrument im praktischen Anwendungsbereich der polizeilichen und justiziellen Zusammenarbeit so nicht vorzufinden. Als Ursachen seien die Mindestbeteiligung von acht Staaten und die einen Kompromiss erschwerende heterogene Interessenlage zu nennen. Vertiefte Zusammenarbeit außerhalb des EU-Rechts sei in verschiedenen Ausprägungen möglich. Die völkerrechtliche Zusammenarbeit mit finaler Ausrichtung diskutierte Böse beispielhaft an der Unterzeichnung des Prümer Vertrags (Mai 2005). Multilaterale Zusammenarbeit und bilaterale Verträge (zum Beispiel die Kooperation zwischen Deutschland und Österreich für grenzüberschreitende Observationen) böten ebenfalls vertragskonforme Möglichkeiten der Kooperation. Die Zusammenarbeit außerhalb des europäischen Vertragswerkes ermögliche notwendigen Spielraum für regionale Spezifikationen, die keinen Bedarf nach einer unionseinheitlichen Regelung hervorrufen. Den Mitgliedstaaten bleibe das Initiativrecht und damit ein höherer Gestaltungsspielraum. Für eine bilaterale beziehungsweise multilaterale Lösung sprächen zudem die zeitlich aufwändige Prozedur für die Umsetzung innerhalb des europäischen Vertragswerkes.

\section{Kerneuropa als eine geeignete Konzeption für die ökonomische Integration?}

Ist ein großer oder ein kleiner Wirtschaftsund Währungsraum optimal für die Umsetzung der ökonomischen Ziele? Dieser Fragestellung, abgeleitet aus den Währungsraumund Außenhandelstheorien, ging Fritz Breuss bei der Bewertung der bisherigen ökonomischen Performanz der Wirtschafts- und Währungsunion nach. Bei Einführung der Gemeinschaftswährung konnte durchaus von 
einer ,großen' Lösung gesprochen werden, denn 12 der 15 Mitgliedstaaten nahmen daran teil. Mit der Osterweiterung habe sich jedoch das Verhältnis der ,Ins' zu den ,Outs ‘ von 12 zu 13 Staaten verschlechtert und weise nun eher Eigenschaften eines Kerneuropas auf. Die bisherigen empirischen Daten lieferten nur die Grundlage für vorsichtige tendenzielle Aussagen über die Vor- und Nachteile einer Teilnahme an dieser Form der differenzierten Integration; insgesamt erscheine die Makroperformanz nicht überwältigend. So seien beispielsweise die Erwartungen hinsichtlich der Steigerung des Bruttoinlandsprodukts und der Einstellung von niedrigen Zinsen sowie Preisen nur teilweise erfüllt worden. Schlüssige Aussagen über handelsstimulierende Effekte der Währungsunion seien (noch) nicht möglich. Allerdings könne trotzdem von Vorteilen für die 12 ,Ins ' gesprochen werden. Insbesondere hätten die Hartwährungsländer gewonnen, da die Weichwährungsländer ihren Vorteil, beruhend auf unfairer Abwertung, nicht mehr realisieren könnten. Breuss sprach sich für die Abkehr von einer differenzierten Integrationslösung für den Währungsraum aus und plädierte für eine rasche Ausweitung der Euro-Zone. Denn nur unter der Bedingung, dass alle Mitgliedstaaten an der wirtschaftlichen Integration partizipieren, könnten die Potentiale dieser voll ausgeschöpft werden. Als problematisch müsse allerdings die Asymmetrie zwischen zentraler Geldpolitik und dezentralen nationalen Fiskalpolitiken bewertet werden. Der hohe Koordinationsaufwand, der mit einer größeren Wirtschafts- und Währungsunion noch steigen würde, stelle die Eignung dieser Struktur als tragfähiges und zukunftsweisendes Konzept in Frage.

In seinem Kommentar regte Richard Senti zum generellen Überdenken der Kopplung einer wirtschaftlichen Integration an die Gemeinschaftswährung an. Er stellte in Frage, dass die Währungsunion tatsächlich Einfluss auf die Im- und Exporte der Europäischen Union habe, wo doch beispielsweise der AuBenhandel viel stärker von (nicht-)tarifären Handelshemmnissen als von Wechselkurs- schwankungen belastet wurde und wird. Insgesamt sah Senti die Währungsunion in erster Linie als ein Instrument zur Ausweitung der politischen Kompetenzen auf der supranationalen Ebene. Die damit verbundene Zentralisierung und Konzentration der Macht bei der Brüsseler Administration führe letztlich jedoch zur Ausschaltung des positiven Wettbewerbs zwischen den Nationalstaaten, der jedoch von den nationalen Politikern nicht unbedingt erwünscht zu sein scheine.

Differenzierte Integration als Lösung oder Last für die Einheitlichkeit in den Außenbeziehungen?

Die Europäische Sicherheits- und Verteidigungspolitik (ESVP) sowie die Gemeinsame Außen- und Sicherheitspolitik (GASP) sind durch eine Vielzahl differenzierter Zusammenarbeiten geprägt: die Europäische Gendarmerie, die Europäische Verteidigungsagentur, die Projekte Eurofighter sowie A400N, die Vorreitergruppe von Großbritannien, Frankreich und Deutschland im Atomstreit mit dem Iran oder aber der Anstoß Polens und Litauens, die kritische Situation in der Ukraine auf die europäische Agenda zu setzen. Mathias Jopp erkannte hier eine Flexibilisierung innerhalb der Union, die es Mitgliedstaaten gemessen an ihren militärischen oder diplomatischen Kapazitäten und ihrem politischen Willen ermögliche, an gemeinsamen Projekten der ESVP/GASP teilzunehmen. Diese differenzierte Integration sei damit die Basis für eine gelungene Zusammenarbeit und keine Ursache für eine mögliche Spaltung. Vielmehr zeigten die aufgeführten Projekte, dass insbesondere im Bereich der GASP der Übergang $\mathrm{zu}$ einer gemeinschaftsrechtlichen Lösung nicht erforderlich sei, denn damit würden letztlich nur politische Handlungen im Primärrecht ex post verankert.

In ihrem Kommentar wies Elisabeth TichyFisslberger auf die Verbesserung der ESVP hin, die in der gemeinschaftlichen Lösung im Entwurf zum Verfassungsvertrag vorgesehen war: Es sollte eine militärische Einheit und 
damit eine gewisse Stärke gegenüber den USA aufgebaut werden. Auch Tichy-Fisslberger registrierte die Vielzahl von politisch bedeutsamen Konstellationen außerhalb des Vertragswerkes, die durchaus eine hohe Effizienz aufweisen. Allerdings richtete sie ihren Blick auch auf die Mitgliedstaaten, die dort nicht eingebunden sind, erkannte dort Unbehagen über die existierenden Informationsund Machtasymmetrien und sah dadurch die Einheit der Union gefährdet.

Stefan Griller stellte die These auf, dass die Handlungsfähigkeit der Union nach außen von den internen Liberalisierungsschritten abhängig sei. In den (wirtschaftlichen) Außenbeziehungen sei die differenzierte Integration allenfalls als Folge interner Differenzierung oder fehlender interner Harmonisierung zu verstehen. Zurückzuführen sei dies auf die komplexe Kompetenzverteilung in der Europäischen Gemeinschaft. So wiesen beispielsweise die Ergebnisse der Verhandlungen im Rahmen des General Agreement on Trade in Services (GATS) verschiedene Integrationsniveaus auf. Die Europäische Union besitze ausschließliche Kompetenzen, zusätzlich würden aber im GATS Fragen behandelt, die die nationalstaatliche handelspolitische Kompetenz berührten. Weiterhin existierten eine Vielzahl von bilateralen und Gruppenabkommen. In der Systematisierung Grillers war differenzierte Integration einerseits als ein $\mathrm{Ne}$ benprodukt interner Differenzierungen $\mathrm{zu}$ verstehen, zum Beispiel in Form der Wirtschafts- und Währungsunion. Andererseits entstehe differenzierte Integration aufgrund zum Teil fehlender oder geteilter Kompetenzen sowie unterschiedlicher Interessen gegenüber beziehungsweise von externen Verhandlungspartnern. Als Beispiele nannte er hier das GATS, das Open-Skies-Abkommen zwischen dem Drittland USA und einzelnen Mitgliedstaaten sowie das Doppelbesteuerungsabkommen. Somit werde deutlich, dass differenzierte Integration in den Außenbeziehungen sehr unterschiedliche Ursachen hat, vom Reflex auf eine fehlende interne Differenzierung bis hin zur fehlenden internen Re- gelung. Ein einheitliches Auftreten gegenüber Dritten könne sie jedoch nicht fördern.

\section{Die verschiedenen Gesichter der differenzier- ten Integration in der Umweltpolitik}

Christian Callies diskutierte, inwiefern differenzierte Integration in Form des Instruments der verstärkten Zusammenarbeit nach Art. 43 bis 45 EUV als geeignet und relevant für die Umweltpolitik bewertet werden könne. Die Besonderheit des Politikfeldes Umweltpolitik ergebe sich aus den unterschiedlichen Handlungsdimensionen (hier von lokal bis international) und -inhalten. Probleme einer gemeinschaftlichen Lösung lägen in den unterschiedlichen Gegebenheiten in den Mitgliedstaaten hinsichtlich der bereits existierenden Standards, der industriellen Struktur und dem damit verbundenen Standortwettbewerb, aber auch in der Betroffenheit von Umweltschäden begründet und daher könne eine klassische Gleichbehandlung nicht wünschenswert sein. Aus der Sicht von Callies ließe sich dieses Dilemma mit der komplementären Flexibilität nach Art. 179 und 95 Abs. 4-5 EGV lösen: Die Europäische Union legt die Richtlinien fest, während die Mitgliedstaaten die Anwendung und Durchsetzung der Richtlinien mit nationaler Auslegung im Sinne einer Schutzverstärkung ausüben. Damit verblieben den Mitgliedstaaten noch ausreichend Kompetenzen und Handlungsspielräume, die in Rückkopplung auf der europäischen Ebene einen Handlungsdruck auslösen könnten. In diesem Sinne würde eine optimale umweltpolitische Zielverwirklichung durch Differenzierung erreicht werden. Dem Instrument der komplementären Flexibilität sei aus Sicht Callies absoluten Vorrang gegenüber der verstärkten Zusammenarbeit einzuräumen, da letztere den Zerfall der Union fördere und zu Verlusten von Freiheiten im Binnenmarkt führe.

Für Alois Leidwein existierten in der Umweltpolitik ein Europa der zwei Geschwindigkeiten und der zwei Gesichter. Ursachen für ers- 
teres seien neben den aufgeführten Punkten von Callies im rechtlichen Hintergrund zu sehen, welcher sich in konkurrierender Zuständigkeit, im Subsidiaritätsprinzip sowie in der Art und Weise der einzelstaatlichen Implementierung niederschlägt. Ein Europa der zwei Gesichter entstehe nach Leidwein aus der Tatsache, dass mit umweltpolitischen Regelungen und Naturschutzbestimmungen innerhalb Europas ein hohes Schutzniveau geschaffen und umweltbelastende Methoden durchaus verteuert wurden. Allerdings setze die europäische Handelspolitik umgekehrt Anreize zur Ausweitung oder Intensivierung der Produktion mit umweltschädigendem Verhalten außerhalb der Europäischen Union. Durch ihre Marktöffnungspolitik würden beispielsweise massive Anreize für die Rodung von Tropenwäldern, die Zerstörung der Primärvegetation und die Intensivierung der Landwirtschaft geschaffen. Dieser Form der differenzierten Integration könne nach Leidwein am besten mit einer Kombination aus ökonomischen Anreizen und staatlichem Zwang zur Durchsetzung nichtmarktfähiger (Umwelt-)Standards begegnet werden. Dafür müsse jedoch erst der ernsthafte politische Wille für das Hineintragen von umweltpolitischen Aspekten in eine europäische und globale Handelspolitik entwickelt werden.

Differenzierte Integration im Dilemma zwischen Impulsgeber und Gefährdung der Einheitlichkeit

Franz Cromme stellte in seinem Beitrag in Frage, dass die differenzierte Integration mit dem Prinzip der Unionstreue vereinbar sei und sie das zwischenstaatliche Gleichgewicht erhalten könne. Als Gegenmaßnahme sei deshalb eine Ausweitung der qualifizierten Mehrheitsentscheidungen voranzutreiben, denn diese verhindere unmittelbar Blockaden und mittelbar die differenzierte Integration als Lösungsweg. Allerdings müsse man sich das ambivalente Verhältnis zwischen Lösungsund Konfliktpotential der differenzierten Integration zum Beispiel anhand jüngster außenpolitischer Ereignisse bewusst werden. Die
Positionierung der Regierungen zum Irakkrieg verdeutliche die Gefahr des nichtgemeinschaftlichen Handelns, während aus dem außervertraglichen Voranschreiten der Avantgarde (,Pralinengipfel ${ }^{\circ}$ ) zukunftsfähige Szenarien resultieren können. Verschiedene Integrationstempi würden aber die Gefahr einer Entwicklung verschiedener Gemeinschaften bergen. Es sei für ein Voranschreiten der Europäischen Union deshalb wichtig, dass ein einheitlicher institutioneller Rahmen existiere, in dem der Erhaltung der demokratischen Legitimation oberste Priorität eingeräumt werde. Dabei könnten durchaus differenzierte Teilräume der Entscheidungsreichweiten vorliegen: Entscheidungen des europäischen Parlaments parallel zu denen der nationalen Parlamente.

Stephan Kux betonte in seinem Kommentar, dass es sich bei der differenzierten Integration lediglich um ein Provisorium und nicht um einen Dauerzustand handele. Daher sei die Gefahr von unterschiedlichen Gemeinschaften real nicht gegeben. Zudem verwies er auf die Tatsache, dass die Mitgliedschaft letztlich nur ein formales Kriterium darstelle, der tatsächliche Bestand der europäischen Union sei hingegen der acquis communautaire, der auch von Nicht-Mitgliedstaaten, beispielsweise der Schweiz, übernommen werde. Diese unterschiedliche Reichweite der Integration sei jedoch als bedenklich einzustufen, da sie Uneinheitlichkeiten der zukünftigen Union manifestiere.

Walter Obwexer konzentrierte seinen Beitrag ebenfalls auf die Anwendung der verstärken Zusammenarbeit nach Art. 43 EUV. Im Zusammenhang mit dem Wettbewerbsrecht wäre diese beispielsweise ein adäquates Instrument für die Einführung einer einheitlichen Bemessungsgrundlage der Körperschaftssteuer. Die Europäische Kommission habe hierzu bereits 2001 einen Vorschlag für die Sicherung fairer Wettbewerbsbedingungen vorgelegt, eine Unterstützung von allen Mitgliedstaaten fand sie bisher nicht. Obwexer stellte der verstärkten Zusammenarbeit in 
Abrede, tatsächlich ein adäquates Instrumentarium im Interesse der Integration und des Wettbewerbsrechts zu sein. Die Erfüllung der rechtlichen Voraussetzungen sei zu umfassend, da das gesamte Primärrecht der Europäischen Union eingehalten und der institutionelle Rahmen beachtet werden müsse.

Daniel Thym betonte in seinem Kommentar die Berücksichtigung der politischen Entscheidungsprozesse. Die alleinige Prüfung der rechtlichen Voraussetzungen für eine verstärkte Zusammenarbeit sei nicht ausreichend, denn politische Entscheidungsträger könnten sich auch bei allen rechtlichen Voraussetzungen gegen eine verstärkte Zusammenarbeit entscheiden. Trotz der Verankerung des Instruments im europäischen Vertragswerk sei es bisher in Binnenmarktsfragen nicht angewendet worden. Thym sah vor allem psychologische Gründe als Ursache. Letztlich bestünde eine gewisse Hürde vor dem Einsatz des Instruments, dass dem Wunsch nach Einheit und Harmonisierung im Recht widerspräche. Zudem fehle der entsprechende Anwendungsbereich: Rechtsakte scheiterten an zumeist inhaltlichen Gründen oder es wurde ein Kompromiss erzielt. Letztlich würden an die verstärkte Zusammenarbeit übergroße Erwartungen gestellt. Sie sei jedoch nur für den Einzelfall gedacht und deshalb keineswegs als ein Einstieg in ein Kerneuropa zu bewerten.

Sektorale Integration: Ein Segen oder Fluch für die Außenbeziehungen?

Anhand der Beziehungen zwischen der Europäischen Union und der Schweiz diskutierte Thomas Cottier die Möglichkeiten sowie Grenzen einer differenzierten Integration mit Drittstaaten. Aus Sicht der Schweiz hätten bilaterale Verträge den entscheidenden Vorteil, ihre Interessen wahren und eine punktuelle Zusammenarbeit mit der Europäischen Union nutzen zu können. Dieser sehr pragmatische Ansatz unterstütze die enge wirtschaftliche Verknüpfung zwischen den Verhandlungspartnern und habe zu einer verbesserten Koordination in der Verkehrspolitik geführt, eine schrittweise Arbeitsmarktöffnung angestoßen und Handelshemmnisse in der Agrarwirtschaft abgebaut. Doch diese positiven Aspekte könnten nicht über die Defizite und Grenzen sektoraler Verträge hinwegtäuschen. So zeige sich beispielsweise, dass die Liberalisierung des Dienstleistungshandels aufgrund seiner hohen Komplexität nicht mittels sektoraler Verhandlungen erreicht werden könne. Die Tatsache, dass die formelle Souveränität der Schweiz nicht angetastet werde, habe die Kehrseite, dass bei der Ausgestaltung von europäischem Recht und europäischen Politiken sowie bei der Anwendung des so genannten nachvollzogenen Rechts die Schweiz keinerlei Mitbestimmungsrechte habe. Die materielle Entscheidung in Brüssel führe zu einem Defizit der demokratischen Legitimität der Schweizer Rechtsordnung. Diese Entwicklungen wiesen auf einen elementaren Zwiespalt in der Schweizer Gesellschaft hin, der symbolisch für Drittstaaten gewertet werden könne: Souveränität und Unabhängigkeit sollen bewahrt werden, Abwehrreaktionen gegen die zunehmende Verlagerung von Entscheidungen auf die europäische sowie internationale Ebene sind die Folge. Gerade diese führten aber dazu, dass Wege der aktiven Mitbestimmung im europäischen Gemeinwesen und Möglichkeiten gegen ,Fremdbestimmung“ verbaut werden.

Aus der Perspektive der Kommentatorin $\mathrm{Ka}$ thrin Blanck stellte sich weniger die Entweder-oder-Frage einer differenzierten Integration oder von konzentrischen Kreisen, als vielmehr nach der Parallelität beider Konzepte. Unter konzentrischen Kreisen würden hier Kooperationen zwischen der Europäischen Union und (Nachbar-)Staaten verstanden, die sich nicht direkt auf die europäischen Verträge beziehen. Allerdings müssten letztere stärker vor dem Hintergrund der strategischen Bedeutung betrachtet werden: Solle ein ,Ring aus Freunden' um die Union gezogen oder mögliche Potenziale zur weiteren Erweiterung der Union eruiert werden? Prinzipiell sei der Kooperationsweg der Schweiz als sukzessive Europäisierung zu werten. In Hinblick 
auf das Innenleben der Europäischen Union plädierte Blanck für den Ausbau der differenzierten Zusammenarbeit, insbesondere mit Blick auf die Bürger und die Distanz zur europäischen Politik.

\section{Unitarisierungstendenzen der differenzierten Integration: Paradoxon oder Realität?}

Peter Christian Müller-Graff sah die differenzierte Integration als ein Abweichen von einer homogenen Integration, da hier der acquis communautaire in seiner Reichweite überschritten und nur von einem Teil der Mitgliedstaaten getragen werde. Die differenzierte Integration könne aus seiner Sicht in die bereits erwähnten verschiedenen Modelle eingeteilt werden. Aber liegt in diesen Modellen der differenzierten Integration eine sprengende Kraft oder eine unitarisierende Wirkung, also die Tendenz zu einer möglichst gleichmäßigen Problemlösung durch die Vereinheitlichung materieller Regelungen? Unter Berücksichtigung des Zeithorizontes sei festzuhalten, dass das Konzept kurzfristig weder unitarisierend angelegt ist noch so wirke. Allerdings sei mittel- bis langfristig durchaus eine unitarisierende Wirkung beobachtbar. Staaten, die zunächst einer differenzierten Integration ferngeblieben seien, wurden mittel- und langfristig einbezogen. Die Betrachtung von fördernden Maßnahmen des Binnenmarktes lasse auf einen Unitarisierungsdruck schließen. Beispielhaft seien die Entwicklungen im Bereich der Arbeitnehmerfreizügigkeit, die letztlich zu einem Wegfall der Personenkontrollen und damit zu einer Neuverständigung in den Bereichen der grenzüberschreitenden Kriminalität führten sowie die Sozialpolitik auf die europäische Agenda brachten. Im Bereich der GASP seien durchaus größere Differenzierungen festzustellen. Doch auch hier führten die Dissonanzen zu einem Unitarisierungsdruck, denn eine Union könne sich auf Dauer eine Vielzahl von Dissonanzen und Bündnissen nicht leisten, wenn sie prioritär eine Einheit bilden wolle. Resümierend hielt Müller-Graff fest, dass die differenzierte Integration im ersten Schritt eine Chance zur Überwindung von Blockaden sei und bedingt durch die unitarisierende Wirkung im zweiten Schritt gegen die Sprengkraft ihrer eigenen Konzeption wirkt.

Christian Joergens stellte in seinem Kommentar heraus, dass sich die verschiedenen Subsysteme der Gesellschaft (Wirtschaft, Kultur, Medien, Politik) mit unterschiedlicher Geschwindigkeit den nationalstaatlichen Grenzen entziehen und somit verschiedenste Probleme aufzeigten. Joergens wies auf die Spannungskräfte zwischen der Umsetzung des europäischen Binnenmarktes und der fehlenden sozialen Homogenität in Europa hin.

In sämtlichen Beiträgen der Tagung wurde zweierlei deutlich: Die differenzierte Integration spielt erstens eine wesentliche Rolle im europäischen Integrationsprozess, nicht nur für die hier betrachteten Politikfelder, sondern auch in den Beziehungen zu Drittstaaten. Es wurde zweitens deutlich, dass dieser Begriff unterschiedlich interpretiert und damit auch konzeptionalisiert wird. Insgesamt wurde erkennbar, dass differenzierte Integration in ihren unterschiedlichsten Auslegungen vor allem als Grundlage für das Fortschreiten der Erweiterung, aber bisher nicht als Ursache für eine Spaltung bewertet werden kann. Darin liegt gerade die Besonderheit ihrer Konzeption: durch die differenzierte Berücksichtigung politischer Interessen kann die europäische Integration und Einheit erhalten sowie gefördert werden. Allerdings verdeutlichten die kritischen Bedenken, dass dem Konzept prinzipiell eine Dynamik unterliegt, die für den europäischen Integrations- und Einigungsprozess bedenklich sein kann und deshalb die Anwendung der differenzierten Integration einer zeitlichen Befristung unterliegen sollte. 